\title{
Chemotherapy induced peripheral neuropathic pain
}

\author{
Hue Jung Park \\ Department of Anesthesiology and Pain Medicine, The Catholic University of Korea College of Medicine, Seoul, Korea
}

Chemotherapy-induced peripheral neuropathy (CIPN) is one of the most serious complications associated with anticancer drugs. CIPN leads to a lower quality of life and dysfunction of the sensory, motor, and autonomic systems, and often causes patients to discontinue chemotherapy. It is usually misdiagnosed and undertreated due to a lack of consensus and unclear pathophysiology, for which many mechanisms have been suggested, including mitochondrial dysfunction, various pain mediators, abnormal spontaneous discharge in A and C fibers, and others. To date, no agents have been shown to effectively prevent CIPN, leading to debate as to the standard protocol. Duloxetine has demonstrated a moderate therapeutic effect against CIPN. Although tricyclic antidepressants (such as nortriptyline or desipramine), gabapentin, and a topical gel containing baclofen $(10 \mathrm{mg})$, amitriptyline HCL (40 mg), and ketamine $(20 \mathrm{mg})$ showed inconclusive results in CIPN trials, these agents are currently considered the best options for CIPN treatment. Therefore, further studies on the pathophysiology and treatment of CIPN are needed. (Korean J Anesthesiol 2014; 67: 4-7)

Key Words: Cancer, Chemotherapy, Pain, Peripheral neuropathy.

\section{Introduction}

Chemotherapy-induced peripheral neuropathy (CIPN) is a frequent, dose-dependent complication of anticancer drugs including platinums, taxanes, epothilones, vinca alkaloids, and newer agents such as bortezomib [1]. It not only leads to dose reduction or discontinuation of treatment but also decreases the quality of life of cancer survivors [2]. CIPN occurs in $~ 20 \%$ of patients given standard doses of chemotherapy and in almost $100 \%$ of patients treated with high doses.

CIPN presents clinically as deficits in sensory, motor, and sometimes autonomic function. Sensory disturbances range from a mild tingling sensation to spontaneous burning pain and hypersensitivity to stimuli. These symptoms often affect both hands and feet and may spread into a 'glove/stocking' distribution. Symptoms are usually symmetrical distally but may be more severe unilaterally. Although dependent on the specific agent, the feet are often affected first. The incidence of CIPN depends on the dose (mainly cumulative) and type of agent; it is more common in patients with preexisting nerve damage either from previous CIPN or from other causes (e.g. diabetes). Symptoms may occur at any time during the course of chemotherapy (seen with paclitaxel), or even after termination (commonly known as "coasting") [3], which refers to neuropathic symptoms

Received: May 8, 2014. Revised: May 12, 2014. Accepted: May 13, 2014.

Corresponding author: Hue Jung Park, M.D., Ph.D., Department of Anesthesiology and Pain Medicine, The Catholic University of Korea College of Medicine, Seoul St. Mary’s Hospital, 222, Banpo-daero, Seocho-gu, Seoul 137-701, Korea. Tel: 82-2-2258-2236, Fax: 82-2-537-1951, E-mail: huejung@catholic.ac.kr

(c) This is an open-access article distributed under the terms of the Creative Commons Attribution Non-Commercial License (http:// creativecommons.org/licenses/by-nc/3.0/), which permits unrestricted non-commercial use, distribution, and reproduction in any medium, provided the original work is properly cited. 
that present after discontinuation of anticancer treatment, and which may continue or worsen over weeks or months. This phenomenon suggests that there is ongoing neuronal damage even after discontinuation of anticancer drugs.

Although the exact pathophysiology of CIPN remains unknown, it is thought that individual chemotherapeutic agents each have a different pathophysiology [4]. Hence, the treatment must also be specific to each chemotherapeutic agent. The pathophysiology and treatment of CIPN are described in this article.

\section{Pathophysiology}

Although the mechanism of development of neuropathic pain due to chemotherapeutic drugs is unknown, various hypotheses have been suggested.

\section{Mitochondrial dysfunction}

Abnormal mitochondrial structure and function are considered a possible etiology of CIPN. The numbers of vacuolated and swollen mitochondria are significantly increased in the $\mathrm{C}$ fibers and myelinated axons of paclitaxel-induced peripheral neuropathy [5]. Mitochondrial caspase activation is essential for chemotherapy-induced apoptosis (vincristine, bortezomib) $[6,7]$. Cisplatin directly inhibits mitochondrial DNA (mtDNA) replication and transcription. Mitochondrial vacuolization and degradation are also observed in cisplatin-treated DRG in vitro and in vivo [8]. Dysregulation of intracellular calcium by mitochondrial dysfunction is involved in bortezomib-induced apoptosis and CIPN $[9,10]$.

Therefore, correction of mitochondrial dysfunction may be considered for prevention or treatment of CIPN. This is supported by several reports that prevention of mitochondrial alterations by acetyl-L-carnitine reduces paclitaxel-induced neuropathic pain [11] and amendment of calcium channel dysregulation decreases pain behaviors $[3,10]$.

\section{Pain mediators}

Changes in many pain mediators in the peripheral nerve, dorsal root ganglion, and spinal cord after anticancer drug treatment have been postulated. These include cytokines, growth factors, ion channels, etc. [4]. Reduction of the nerve growth factor level is correlated with the degree of cisplatin-induced peripheral neuropathy [12]. Mitogen-activated protein kinases and extracellular signal-related kinases may play important roles in CIPN; activation of these kinases is involved in chemotherapyinduced apoptosis [13].

The TNF- $\alpha$ level is increased in the sciatic nerves and spinal cords of a CIPN animal model [14,15]. Administration of TNF- $\alpha$ neutralizing antibodies reduced vincristine-induced tactile hyperalgesia significantly [15]. When IL-1 $\beta$ release was blocked in the CIPN rat model, mechanical hyperalgesia was also reduced [16]. The IL-6 level was increased in the sciatic nerve and DRG of CIPN mice, and administration of IL- 6 antibodies to the sciatic nerve reduced tactile hyperalgesia significantly. Additionally, chemotherapy-induced tactile hyperalgesia was less common in IL-6 KO mice than in wild-type mice [17]. These results suggest that proinflammatory cytokines such as TNF- $\alpha$, IL-1, and IL-6 are implicated in CIPN [4].

Fos expression was activated significantly in the superficial, intermediate, and deep layers of the spinal cord in a CIPN animal model [18]. Also, Fos protein was distributed more widely in the dorsal horn of a CIPN animal model than in a nerve injury animal model [19].

\section{Abnormal spontaneous discharge in $\mathrm{A}$ and $\mathrm{C}$ fibers}

Peripheral and central sensitization may be involved in the development of CIPN. Abnormal spontaneous activity in $A \beta$, $\mathrm{A} \delta$, and $\mathrm{C}$ fibers has been observed in vincristine-, paclitaxel-, oxaliplatin-, and bortezomib-induced peripheral neuropathy [20,21]. Moreover, C-fiber nociceptors showed significant hyperresponsiveness to suprathreshold tactile and thermal stimuli in a CIPN animal model [22].

\section{Others}

Paclitaxel-, oxaliplatin-, and vincristine-induced peripheral neuropathy is caused by decreased numbers of intraepidermal sensory fibers and increased numbers of epidermal Langerhans cells [23], and resembles a traumatic-nerve-injury-induced neuropathic pain model (CCI, sciatic nerve transection). The CNS has also been shown to be involved in CIPN both directly and indirectly [24].

\section{The mechanisms of CIPN may differ from those of other types of neuropathic pain}

First, hyperalgesia to mechanical and cold stimuli usually manifests in CIPN. However, heat hyperalgesia is nearly absent in a model of cisplatin-, paclitaxel-, and vincristine-induced CIPN [25]. Heat hyperalgesia is observed frequently in a model of neuropathic pain due to traumatic nerve injury (e.g. CCI, SNL, etc.).

Second, the abnormal spontaneous discharge in $\mathrm{A} \beta, \mathrm{A} \delta$, and $C$ fibers does not resemble the pattern seen in nerve injuryinduced neuropathic pain models, as only 2-3 spikes/s are observed in CIPN rather than the 20-30 spikes/s in nerve-injuryinduced neuropathic pain models [20]. 
Third, CIPN shares several mediators and processes with other types of neuropathic pain. Nevertheless, the efficacy of antineuropathic drugs differ [26].

These discrepancies indicate that the pathophysiology of CIPN may differ from that of nerve-injury-induced neuropathic pain.

\section{Treatment and Prevention}

Therapeutic strategies should not hinder the anticancer effects of chemotherapy. Although some data suggest that certain substances may prevent CIPN [2], no treatments have been demonstrated to prevent CIPN effectively. Thus, no accepted standard protocol exists [27].

Pregabalin may be used to decrease neuropathy after oxaliplatin treatment [28]. Duloxetine showed modest analgesic efficacy in CIPN (paclitaxel- and oxaliplatin-induced) patients compared with a placebo in a randomized clinical trial $[29,30]$. These data support a moderate recommendation for CIPN treatment [27]. Among antidepressants, venlafaxine has been shown to reduce the incidence of CIPN, albeit with some side effects [31]. Cannabinoids may be a new treatment option for CIPN, and should be evaluated in robust RCTs [32]. Although tricyclic antidepressants (such as nortriptyline or desipramine), gabapentin, and a topical gel containing baclofen (10 mg), amitriptyline HCL (40 mg), and ketamine (20 mg) yielded inconclusive data in CIPN trials, these agents may constitute the remaining CIPN treatment options [27].

Neuroprotectants exhibit only very weak efficacy in terms of preventing CIPN [33], but nutraceuticals, as alternatives to pharmacotherapeutics, have shown more promising results in the treatment and prevention of CIPN [34]. Vitamin E has been used to reduce cisplatin- and paclitaxel-induced neuropathic pain [35], while menthol (a TRPM8 channel activator) has analgesic effects in carboplatin- and bortezomib-induced neuropathy [36,37]. Drugs that increase mitochondrial function have shown some efficacy in laboratory studies and may be eligible to enter clinical trials regarding the treatment and prevention of CIPN $[11,38,39]$.

\section{Conclusions}

Anticancer chemotherapeutics can induce painful peripheral neuropathy. Symptoms range widely and can involve the sensory, motor, and autonomic systems. However, CIPN is under-assessed and undertreated and its diagnosis is somewhat complicated by the lack of a consensus on its pathophysiology and presentation. Although limited reliable evidence regarding the appropriate treatment for this condition exists, it is based on current neuropathic pain guidelines. Further studies of the differences between the pathophysiology of CIPN and those of other neuropathic pain conditions may lead to development of more effective treatment modalities. Additionally, therapeutic strategies for the management of CIPN must be validated in large-scale RCTs to satisfy the demands of evidenced-based medicine.

\section{Acknowledgments}

The author wishes to acknowledge the financial support of the Catholic Medical Center Research Foundation made in the program year of 2013 .

\section{References}

1. Quasthoff S, Hartung HP. Chemotherapy-induced peripheral neuropathy. J Neurol 2002; 249: 9-17.

2. Wolf S, Barton D, Kottschade L, Grothey A, Loprinzi C. Chemotherapy-induced peripheral neuropathy: prevention and treatment strategies. Eur J Cancer 2008; 44: 1507-15.

3. Flatters SJ, Bennett GJ. Ethosuximide reverses paclitaxel- and vincristine-induced painful peripheral neuropathy. Pain 2004; $109: 150-61$.

4. Cata JP, Weng HR, Lee BN, Reuben JM, Dougherty PM. Clinical and experimental findings in humans and animals with chemotherapyinduced peripheral neuropathy. Minerva Anestesiol 2006; 72: 151-69.

5. Flatters SJ, Bennett GJ. Studies of peripheral sensory nerves in paclitaxel induced painful peripheral neuropathy: evidence for mitochondrial dysfunction. Pain 2006; 122: 245-57.

6. Mitsiades N, Mitsiades CS, Poulaki V, Chauhan D, Fanourakis G, Gu X, et al. Molecular sequelae of proteasome inhibition in human multiple myeloma cells. Proc Natl Acad Sci U S A 2002; 99: 14374-9.

7. Joseph EK, Levine JD. Caspase signalling in neuropathic and inflammatory pain in the rat. Eur J Neurosci 2004; 20: 2896-902.

8. Podratz JL, Knight AM, Ta LE, Staff NP, Gass JM, Genelin K, et al. Cisplatin induced mitochondrial DNA damage in dorsal root ganglion neurons. Neurobiol Dis 2011; 41: 661-8.

9. Landowski TH, Megli CJ, Nullmeyer KD, Lynch RM, Dorr RT. Mitochondrial-mediated disregulation of Ca2+ is a critical determinant of Velcade (PS-341/bortezomib) cytotoxicity in myeloma cell lines. Cancer Res 2005; 65: 3828-36.

10. Siau C, Bennett GJ. Dysregulation of cellular calcium homeostasis in chemotherapy-evoked painful peripheral neuropathy. Anesth Analg 2006; 102: 1485-90. 
11. Jin HW, Flatters SJ, Xiao WH, Mulhern HL, Bennett GJ. Prevention of paclitaxel-evoked painful peripheral neuropathy by acetyl-L-carnitine: Effects on axonal mitochondria, sensory nerve fiber terminal arbors, and cutaneous Langerhans cells. Exp Neurol 2008; 210: 229-37.

12. Cavaletti G, Pezzoni G, Pisano C, Oggioni N, Sala F, Zoia C, et al. Cisplatin-induced peripheral neurotoxicity in rats reduces the circulating levels of nerve growth factor. Neurosci Lett 2002; 322: 103-6.

13. Scuteri A, Galimberti A, Maggioni D, Ravasi M, Pasini S, Nicolini G, et al. Role of MAPKs in platinum-induced neuronal apoptosis. Neurotoxicology 2009; 30: 312-9.

14. Muthuraman A, Singh N, Jaggi AS. Protective effect of Acorus calamus L. in rat model of vincristine induced painful neuropathy: an evidence of anti-inflammatory and anti-oxidative activity. Food Chem Toxicol 2011; 49: 2557-63.

15. Kiguchi N, Maeda T, Kobayashi Y, Kishioka S. Up-regulation of tumor necrosis factor-alpha in spinal cord contributes to vincristineinduced mechanical allodynia in mice. Neurosci Lett 2008; 445: 140-3.

16. Honore P, Donnelly-Roberts D, Namovic MT, Hsieh G, Zhu CZ, Mikusa JP, et al. A-740003 [N-(1-\{[(cyanoimino)(5-quinolinylamino) methyl]amino\}-2,2-dimethylpropyl)-2-(3,4-dimethoxyphenyl)acetamide], a novel and selective P2X7 receptor antagonist, dose-dependently reduces neuropathic pain in the rat. J Pharmacol Exp Ther 2006; 319: 1376-85.

17. Kiguchi N, Maeda T, Kobayashi Y, Kondo T, Ozaki M, Kishioka S. The critical role of invading peripheral macrophage-derived interleukin-6 in vincristine-induced mechanical allodynia in mice. Eur J Pharmacol 2008; 592: 87-92.

18. Haselman MA. Dexmedetomidine: a useful adjunct to consider in some high-risk situations. AANA J 2008; 76: 335-9.

19. Coggeshall RE. Fos, nociception and the dorsal horn. Prog Neurobiol 2005; 77: 299-352.

20. Xiao WH, Bennett GJ. Chemotherapy-evoked neuropathic pain: abnormal spontaneous discharge in A-fiber and C-fiber primary afferent neurons and its suppression by acetyl-L-carnitine. Pain 2008; 135: 262-70.

21. Cata JP, Weng HR, Burton AW, Villareal H, Giralt S, Dougherty PM. Quantitative sensory findings in patients with bortezomib-induced pain. J Pain 2007; 8: 296-306.

22. Tanner KD, Reichling DB, Levine JD. Nociceptor hyper-responsiveness during vincristine-induced painful peripheral neuropathy in the rat. J Neurosci 1998; 18: 6480-91.

23. Siau C, Xiao W, Bennett GJ. Paclitaxel- and vincristine-evoked painful peripheral neuropathies: loss of epidermal innervation and activation of Langerhans cells. Exp Neurol 2006; 201: 507-14.

24. Bennett GJ. Pathophysiology and animal models of cancer-related painful peripheral neuropathy. Oncologist 2010; 15 Suppl 2: 9-12.

25. Park HJ, Stokes JA, Pirie E, Skahen J, Shtaerman Y, Yaksh TL. Persistent hyperalgesia in the cisplatin-treated mouse as defined by threshold measures, the conditioned place preference paradigm, and changes in dorsal root ganglia activated transcription factor 3: the effects of gabapentin, ketorolac, and etanercept. Anesth Analg 2013; 116: 224-31.

26. Farquhar-Smith P. Chemotherapy-induced neuropathic pain. Curr Opin Support Palliat Care 2011; 5: 1-7.

27. Hershman DL, Lacchetti C, Dworkin RH, Lavoie Smith EM, Bleeker J, Cavaletti G, et al. Prevention and management of chemotherapyinduced peripheral neuropathy in survivors of adult cancers: american society of clinical oncology clinical practice guideline. J Clin Oncol 2014; 32: 1941-67.

28. Saif MW, Syrigos K, Kaley K, Isufi I. Role of pregabalin in treatment of oxaliplatin-induced sensory neuropathy. Anticancer Res 2010 ; 30 : 2927-33.

29. Smith EM, Pang H, Cirrincione C, Fleishman S, Paskett ED, Ahles T, et al. Alliance for Clinical Trials in Oncology. Effect of duloxetine on pain, function, and quality of life among patients with chemotherapy-induced painful peripheral neuropathy: a randomized clinical trial. JAMA 2013; 309: 1359-67.

30. Smith EM, Pang H. Therapy for chemotherapy-induced peripheral neuropathy--in reply. JAMA 2013; 310: 538.

31. Özdogan M, Samur M, Bozcuk H, Aydin H, Çoban E, Savas B. Venlafaxine for treatment of chemotherapy-induced neuropathic pain. Turk J Cancer 2004; 34: 110-3.

32. Lynch ME, Cesar-Rittenberg P, Hohmann AG. A double-blind, placebo-controlled, crossover pilot trial with extension using an oral mucosal cannabinoid extract for treatment of chemotherapyinduced neuropathic pain. J Pain Symptom Manag 2014; 47: 166-73.

33. Argyriou AA, Bruna J, Marmiroli P, Cavaletti G. Chemotherapy induced peripheral neurotoxicity (CIPN): an update. Crit Rev Oncol Hematol 2012; 82: 51-77.

34. Schloss JM, Colosimo M, Airey C, Masci PP, Linnane AW, Vitetta L. Nutraceuticals and chemotherapy induced peripheral neuropathy (CIPN): a systematic review. Clin Nutr 2013; 32: 888-93.

35. Argyriou AA, Chroni E, Koutras A, Ellul J, Papapetropoulos S, Katsoulas G, et al. Vitamin E for prophylaxis against chemotherapy-induced neuropathy: a randomized controlled trial. Neurology 2005; 64: 26-31.

36. Storey DJ, Colvin LA, Mackean MJ, Mitchell R, Fleetwood-Walker SM, Fallon MT. Reversal of dose-limiting carboplatin-induced peripheral neuropathy with TRPM8 activator, menthol, enables further effective chemotherapy delivery. J Pain Symptom Manage 2010; 39: e2-4.

37. Colvin LA, Johnson PR, Mitchell R, Fleetwood-Walker SM, Fallon M. From bench to bedside: a case of rapid reversal of bortezomibinduced neuropathic pain by the TRPM8 activator, menthol. J Clin Oncol 2008; 26: 4519-20.

38. Xiao WH, Zheng FY, Bennett GJ, Bordet T, Pruss RM. Olesoxime (cholest-4-en-3-one, oxime): analgesic and neuroprotective effects in a rat model of painful peripheral neuropathy produced by the chemotherapeutic agent, paclitaxel. Pain 2009; 147: 202-9.

39. Flatters SJ, Xiao WH, Bennett GJ. Acetyl-L-carnitine prevents and reduces paclitaxel-induced painful neuropathy. Neurosci Lett 2006; $397: 219-23$. 